\title{
Inter-arm blood pressure difference in type 2 diabetes: a barrier to effective management?
}

\author{
Christopher E Clark, Colin J Greaves, Philip H Evans, Andy Dickens and John L Campbell
}

\begin{abstract}
Background

Previous studies have identified a substantial prevalence of a blood pressure difference between arms in various populations, but not patients with type 2 diabetes. Recognition of such a difference would be important as a potential cause of underestimation of
\end{abstract} blood pressure.

Aim

To measure prevalence of an inter-arm blood pressure difference in patients with type 2 diabetes, and to estimate how frequently blood pressure measurements could be erroneously underestimated if an inter-arm difference is unrecognised.

Design of study

Cross-sectional study.

Setting

Five surgeries covered by three general practices,

Devon, England.

Method

Patients with type 2 diabetes underwent bilateral simultaneous blood pressure measurements using a validated protocol. Mean blood pressures were calculated for each arm to derive mean systolic and diastolic differences, and to estimate point prevalence of predefined magnitudes of difference.

Results

A total of 101 participants were recruited. Mean age was 66 years (standard deviation [SD] = 13.9 years); $59 \%$ were male, and mean blood pressure was $138 / 79 \mathrm{mmHg}$ ( $\mathrm{SD}=15 / 10 \mathrm{mmHg}$ ). Ten participants (10\%; $95 \%$ confidence interval $[\mathrm{Cl}]=4$ to 16$)$ had a systolic inter-arm difference $\geq 10 \mathrm{mmHg} ; 29(29 \%$; $95 \%$ $\mathrm{Cl}=20$ to 38) had a diastolic difference $\geq 5 \mathrm{mmHg}$; and three $(3 \% ; 95 \% \mathrm{Cl}=0$ to 6$)$ a diastolic difference $\geq 10 \mathrm{mmHg}$. No confounding variable was observed to account for the magnitude of an inter-arm difference.

\section{Conclusion}

A systolic inter-arm difference $\geq 10 \mathrm{mmHg}$ was observed in $10 \%$ of patients with diabetes. Failure to recognise this would misclassify half of these as normotensive rather than hypertensive using the lowerreading arm. New patients with type 2 diabetes should be screened for an inter-arm blood pressure difference. Keywords

blood pressure measurement; inter-arm difference; peripheral vascular disease; type 2 diabetes mellitus.

\section{INTRODUCTION}

In type 2 diabetes accurate measurement of blood pressure is a key component of the regular review, and central to the management of cardiovascular risk. ${ }^{1}$ Previous studies by the current authors ${ }^{2-4}$ have identified a reproducible difference in the blood pressure between arms as an important potential cause of error if not recognised and if measurements are not standardised on the higher arm, as guidelines advise. ${ }^{5}$ A systematic review of the literature on this inter-arm difference showed a weighted mean prevalence for a systolic inter-arm difference $\geq 10 \mathrm{mmHg}$ of $20 \%$ (95\% confidence interval $[\mathrm{Cl}]=$ 18 to $21 \%$ ) in hospital-based studies of in- and outpatients. No primary care study met the inclusion criteria and no study that reported a prevalence in patients with diabetes, either post hoc or by design, was identified. ${ }^{6}$

In their study of 492 participants presenting for cardiac catheterisation, English et al reported a $7 \%$ prevalence of $>50 \%$ left-subclavian stenosis (the presumed cause of an inter-arm difference) in patients with diabetes, compared with $12 \%$ and $2 \%$ in the presence and absence of peripheral vascular disease respectively. ${ }^{7}$ In Lane et al's hospital

CE Clark, MSc, FRCP, FRCGP, clinical academic fellow; CJ Greaves, PhD, CPsychol, senior research fellow;

PH Evans, MPhil, FRCGP, senior clinical research fellow; A Dickens, MSc, research fellow; JL Campbell, MD, MRCGP, professor of general practice and primary care, Institute of Health Service Research, Peninsula College of Medicine and Dentistry, Exeter, Devon.

Address for correspondence

Dr Christopher Clark, Primary Care Research Group, Institute of Health Service Research, Peninsula College of Medicine and Dentistry, Smeall Building, St Luke's Campus, Magdalen Rd, Exeter, Devon, EX1 2LU.

Email: christopher.clark@pms.ac.uk

Submitted: 2 July 3008; Editor's response: 16 September 2008; final acceptance: 30 October 2008.

OBritish Journal of General Practice.

This article was originally online first on $30 \mathrm{Apr} 2009$. Cite this article as: Br J Gen Pract 2009; 59: 428-432. Advance online publication. DOI: 10.3399/bjgp09X420752 
prevalence study $15 \%$ of patients had diabetes. An overall prevalence of $20 \%$ systolic inter-arm difference $\geq 10 \mathrm{mmHg}$ and $11 \%$ diastolic inter-arm difference $\geq 10 \mathrm{mmHg}$ was reported; no separate diabetic inter-arm difference prevalences were reported, but diabetes was not a significant predictor of an inter-arm difference. ${ }^{8}$

One Dutch study recently reported a prevalence of systolic or diastolic inter-arm difference $>10 \mathrm{mmHg}$ for $9 \%$ of 169 general practice patients with type 2 diabetes. ${ }^{9}$ Reproducibility of an inter-arm difference $>10 \mathrm{mmHg}$ after 12 months was poor. However, these authors adopted a retrospective comparison of two simultaneous pairs of blood pressures with a previous single pair of sequential measurements (personal communication, N Kleefstra, 2008). Previous studies have overestimated inter-arm difference prevalence due to poor methodology, most commonly by employing a non-simultaneous technique of bilateral arm blood pressure measurement. ${ }^{6}$ Therefore, the current study was designed to record simultaneous bilateral blood pressure measurements using a previously validated, robust technique ${ }^{4}$ in patients with type 2 diabetes in primary care. This estimated the prevalence of an inter-arm difference and how often blood pressure might be significantly underestimated if the inter-arm difference is not recognised.

\section{METHOD}

From October 2005 to July 2007 all patients with type 2 diabetes, identified from the diabetes registers in three practices covering five surgeries (combined population 16 000) in Devon, England, were invited to undergo bilateral blood pressure measurement during routine assessment for diabetic review. Recruitment was by letter, with a single telephone reminder to non-responders.

For the study, blood pressure was measured simultaneously in both arms with two automated sphygmomanometers using a previously piloted protocol. ${ }^{4}$ In brief, this involved the participant sitting quietly for 5 minutes. Two pairs of bilateral blood pressure measurements were then obtained by simultaneous activation of two automated sphygmomanometers (Omron 711, Omron Healthcare), the cuffs were then swapped to the contralateral arms, and two further pairs of blood pressure readings obtained. The order of application of machines to arms was randomised by means of a random number table. Data collection sheets were completed with demographic data (that is, age, sex, body mass index [BMI], waist and hip measurements, and smoking status) and samples were collected for total cholesterol, glycosylated haemoglobin ( $\mathrm{HbA} 1 \mathrm{c})$, creatinine, and presence of microalbuminuria. In

\section{How this fits in}

Accurate measurement of blood pressure is vital for risk factor management in type 2 diabetes. Failure to recognise a reproducible difference in pressures between arms could delay or prevent diagnosis of hypertension if the lowerreading arm is recorded. This study demonstrates that a $10 \mathrm{mmHg}$ or greater difference in systolic pressures is seen in $10 \%$ of patients with type 2 diabetes, and that half of these participants would be misclassified as normotensive using current targets. Initial assessment of new patients with diabetes should therefore include bilateral blood pressure measurement to standardise future readings to the higher arm.

addition a brief two-question assessment of the acceptability of this technique of blood pressure measurement was employed. The information was collated and analysed with SPSS (version 15).

Mean systolic and diastolic pressures were calculated for each arm (for each individual) and subtracted to derive inter-arm systolic and diastolic blood pressure differences. The prevalence of interarm differences greater than $10 \mathrm{mmHg}$ was then derived using simple descriptive statistics. To explore differences in characteristics between attenders and non-attenders, and between participants with or without an inter-arm difference, Student's $t$-tests for continuous data and $\chi^{2}$ or Fisher's exact test for categorical data were used.

Based on previous findings, calculations suggested that a sample size of 94 would have a power of $90 \%$ to detect a $10 \%$ prevalence of interarm difference with $95 \% \mathrm{Cl}^{4}$

\section{RESULTS}

Following invitations to 170 potential participants, 101 patients with type 2 diabetes (59\% acceptance) agreed to participate (mean age 66 years, standard deviation $[\mathrm{SD}]=13.9$ years]; 59\% male). Mean blood pressure was 138/79 $\mathrm{mmHg}(\mathrm{SD}=15 / 10 \mathrm{mmHg}$ ), and a previous medical history of angina was reported by $19(19 \%)$, myocardial infarction by six $(6 \%)$, stroke by six $(6 \%)$, and peripheral vascular disease by two (2\%). Attenders were not significantly different from non-attenders in respect of age, sex, smoking status, $\mathrm{BMI}, \mathrm{HbA1c}$, total cholesterol, presence of microalbuminuria, or systolic blood pressure when compared using $t$-test or $\chi^{2}$ statistics as appropriate (Table 1, Table 2). However, non-attenders did have a significantly lower last-recorded diastolic blood pressure $(73 \mathrm{mmHg}$ versus $79 \mathrm{mmHg}$, mean difference $=7[95 \% \mathrm{Cl}=4$ to 10$], P<0.001)$.

Ten participants (10\%; $95 \% \mathrm{Cl}=4$ to 16$)$ had a systolic inter-arm difference $\geq 10 \mathrm{mmHg}$ (Figure 1), 29 (29\%; $95 \% \mathrm{Cl}=20 \%$ to $38 \%$ ) had a diastolic difference $\geq 5 \mathrm{mmHg}$ and three $(3 \% ; 95 \% \mathrm{Cl}=0$ to 6$)$ 
Table 1. Comparison of attenders and non-attenders, continuous variables.

\begin{tabular}{|c|c|c|c|c|c|c|c|}
\hline & \multicolumn{2}{|c|}{ Attenders } & \multicolumn{2}{|c|}{ Non-attenders } & \multirow{2}{*}{$\begin{array}{c}\text { Mean } \\
\text { difference }\end{array}$} & \multirow[b]{2}{*}{$95 \% \mathrm{Cl}$} & \multirow[b]{2}{*}{$P$-value } \\
\hline & $n$ & Mean & $n$ & Mean & & & \\
\hline Last recorded systolic $\mathrm{BP}, \mathrm{mmHg}$ & 101 & 137.6 & 67 & 133.2 & 4.4 & -0.7 to 9.4 & 0.093 \\
\hline Last recorded diastolic $\mathrm{BP}, \mathrm{mmHg}$ & 101 & 79.2 & 67 & 72.5 & 6.7 & 3.7 to 9.7 & $<0.001$ \\
\hline$\underline{\mathrm{BMI}}$ & 100 & 29.9 & 67 & 29.4 & 0.5 & -1.4 to 2.4 & 0.60 \\
\hline $\mathrm{HbA1c}$ & 56 & 7.3 & 67 & 7.3 & 0.0 & -0.4 to 0.4 & 0.95 \\
\hline Total cholesterol & 92 & 4.2 & 67 & 4.0 & 0.2 & -0.1 to 0.4 & 0.31 \\
\hline Age, years & 93 & 66.0 & 66 & 66.4 & -0.4 & -4.7 to 3.8 & 0.84 \\
\hline
\end{tabular}

$B P=$ blood pressure $. B M I=$ body mass index. $\mathrm{HbA1C}=$ glycosylated haemoglobin

had a diastolic inter-arm difference $\geq 10 \mathrm{mmHg}$ (Figure 2). The presence of a systolic inter-arm difference $\geq 10$ $\mathrm{mmHg}$ or a diastolic inter-arm difference $\geq 5 \mathrm{mmHg}$ was not related to differences in age, sex, smoking status, BMI, waist circumference, $\mathrm{HbA} 1 \mathrm{c}$, total cholesterol, serum creatinine, presence of microalbuminuria, or absolute levels of systolic or diastolic blood pressure when compared using $t$-test and $\chi^{2}$ or Fisher's exact test as appropriate to the data.

The presence of a systolic or diastolic inter-arm difference was not related to a previous medical history of peripheral vascular disease, stroke, or myocardial infarction. The presence of a diastolic inter-arm difference was not related to a previous medical history of angina. However, a systolic interarm difference $\geq 5 \mathrm{mmHg}$ or $\geq 8 \mathrm{mmHg}$ was associated with an increased prevalence of a previous medical history of angina: $63 \%$ with $\geq 5 \mathrm{mmHg}$ versus $29 \%$ without $(\mathrm{OR}=4.1,95 \% \mathrm{Cl}=$ 1.5 to $11.8, P<0.01)$ and $42 \%$ with $\geq 8 \mathrm{mmHg}$ versus $13 \%$ without $(\mathrm{OR}=4.7,95 \% \mathrm{Cl}=1.5$ to 14.2 , $P<0.01)$. Using the current NICE target blood pressure of $140 / 80 \mathrm{mmHg}$, six of the 10 participants with a systolic inter-arm difference of $\geq 10 \mathrm{mmHg}$ would be misclassified as normotensive using the lower-pressure arm, as would five of the 10 using the more stringent target of $130 / 80 \mathrm{mmHg}$ suggested for those with kidney, eye, or cerebrovascular damage..$^{10}$

Of 100 participants who responded, 99 (99\%) stated that they would be happy to have blood pressure assessed by this technique again in the

Table 2. Comparison of attenders and non-attenders, categorical variables.

\begin{tabular}{|c|c|c|c|c|c|c|}
\hline & \multicolumn{2}{|c|}{ Attenders } & \multicolumn{2}{|c|}{ Non attenders } & \multirow[b]{2}{*}{$\chi^{2}$} & \multirow[b]{2}{*}{$P$-value } \\
\hline & $n$ & $\%$ & $n$ & $\%$ & & \\
\hline Male sex & $61 / 101$ & 60.4 & $35 / 67$ & 52.2 & 0.8 & 0.43 \\
\hline Current smoker & $10 / 101$ & 9.9 & $14 / 67$ & 20.9 & 4.2 & 0.05 \\
\hline Ex-smoker & $55 / 101$ & 54.5 & $28 / 67$ & 41.8 & 2.2 & 0.16 \\
\hline Never smoker & $36 / 101$ & 35.6 & $24 / 67$ & 35.8 & $<0.001$ & 1.00 \\
\hline Microalbuminuria present & $19 / 83$ & 22.9 & $10 / 67$ & 14.9 & 1.5 & 0.30 \\
\hline
\end{tabular}

future, and only one $(1 \%)$ reported that they would not, due to the discomfort experienced.

\section{DISCUSSION}

\section{Summary of main findings}

This study reports the first UK estimated prevalence of an inter-arm blood pressure difference in a primary care sample of patients with type 2 diabetes. A systolic inter-arm difference $\geq 10 \mathrm{mmHg}$ was observed in $10 \%$ of patients with diabetes, a diastolic inter-arm difference $\geq 5 \mathrm{mmHg}$ in $29 \%$, and a diastolic inter-arm difference $\geq 10 \mathrm{mmHg}$ was observed in $3 \%$. The observed inter-arm difference was unrelated to absolute blood pressure or other potential confounding variables, and the measurement technique was found to be acceptable to patients.

\section{Strengths and limitations of the study}

This study recruited patients with diabetes from urban and rural surgeries in Devon. The simultaneous measurement protocol was adapted from Gould et $a^{111}$ and minimises bias. ${ }^{12}$ The study was previously piloted, ${ }^{4}$ and participants in the current study reported a high acceptance rate for the procedure.

Although analysis of non-responders indicated that the sample recruited was representative of the total diabetic population in these surgeries, the recruitment rate of only $59 \%$ was lower than anticipated. This was due to limited nursing resources to maintain recruitment and follow up nonresponders. An unforeseen barrier was the difficulty of identifying clinical nursing time within the working day to gather the data, which added a maximum of 10 minutes per participant to routine clinical reviews.

However, the actual sample size was greater than that projected and thus the study was adequately powered to derive the point prevalence estimates reported. Comparison of attenders and nonattenders showed no significant differences except a $7 \mathrm{mmHg}$ higher diastolic blood pressure in attenders. Inter-arm differences were not related to absolute blood pressure levels.

Ethnic minority representation is low in Devon, 
particularly in rural areas, and participants were predominantly white; therefore, caution may be required in extrapolating these findings to other ethnic groupings.

\section{Comparison with existing literature}

Previous secondary care studies have suggested a wide range of inter-arm difference prevalences in varied populations. Only one previous primary care estimate has been published, from the Netherlands, which had similar findings. That study reported a similar prevalence to that reported in the current study, namely $9 \%$ systolic or diastolic inter-arm difference $>10 \mathrm{mmHg}$ with only two pairs of simultaneous measurements, or $33 \%$ if only a single pair of sequential measurements were taken. ${ }^{9}$ This confirms a previous review by the current authors that prevalence of an inter-arm difference may be overestimated by poor methodology and the use of a non-simultaneous measurement technique. ${ }^{6}$

There is evidence to associate the inter-arm difference with peripheral vascular disease $e^{4,7}$ and increased cardiovascular risk, ${ }^{3,13,14}$ and an association was found in this study of a systolic inter-arm difference $\geq 5 \mathrm{mmHg}$ or $\geq 8 \mathrm{mmHg}$ with angina. Other vascular conditions (stroke, myocardial infarction, and peripheral vascular disease) were not associated with the inter-arm difference but numbers reporting these conditions were much fewer.

\section{Implications for clinical practice and future research}

The observation of a systolic inter-arm difference of $\geq 10 \mathrm{mmHg}$ in $10 \%$ of patients with diabetes implies that systolic blood pressure could be underestimated by this amount for 1 in 20 cases (5\% of diabetic consultations) by chance if the higher-pressure arm has not been identified. Systolic blood pressure is an important risk factor for diabetes-related mortality ${ }^{1}$ and the majority of participants with a systolic interarm difference $\geq 10 \mathrm{mmHg}$ would have been misclassified as normotensive against current guidelines using the lower-pressure arm. ${ }^{10}$ Therefore, it is proposed that blood pressure should be measured in both arms when assessing new patients with diabetes to promote effective care by standardising blood pressure readings to the higherpressure arm. The study protocol, while minimising bias, did increase the consultation time. A simpler sequential approach measuring one arm then the other could, however, easily be adopted for screening in practice. Repeated or simultaneous measurements could then be reserved for further consultations if needed to confirm a difference. ${ }^{15}$

Further studies are underway to confirm the current findings in a larger sample and to address doubts over

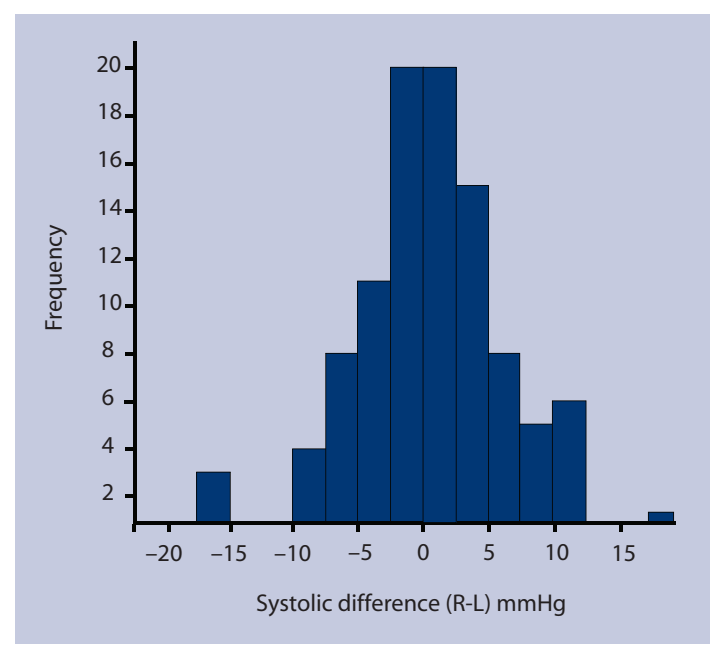

Figure 1. Distribution of systolic inter-arm blood pressure difference in 101 patients with type 2 diabetes.

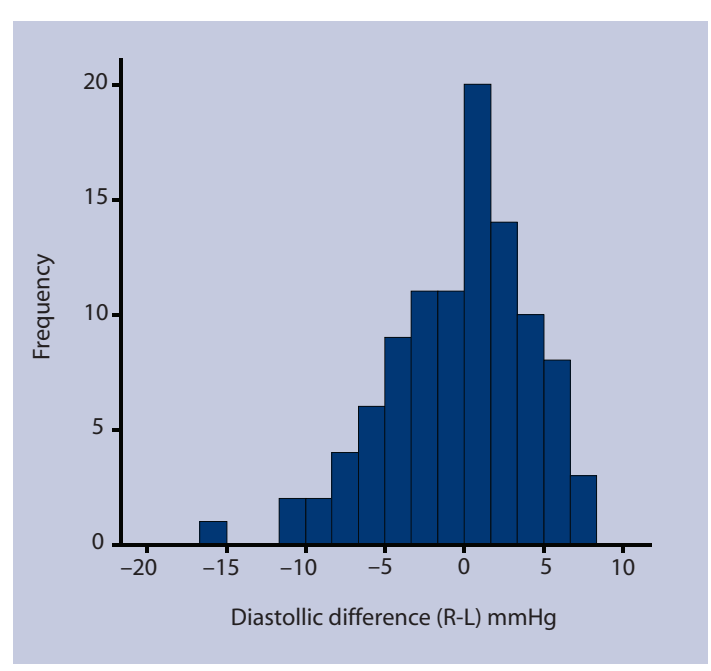

Figure 2. Distribution of diastolic inter-arm blood pressure difference in 101 patients with type 2 diabetes. the reproducibility of an inter-arm difference over time. ${ }^{9,16}$ Further work is needed to establish whether published associations of an inter-arm difference with cardiovascular and peripheral vascular disease can also be demonstrated in patients with type 2 diabetes. Work is also needed to identify what role the detection of an inter-arm difference may play in predicting the presence of peripheral vascular disease to facilitate estimation of cardiovascular risk and targeting of interventions in this growing population. ${ }^{17}$

A systolic inter-arm difference of $\geq 10 \mathrm{mmHg}$ is observed in $10 \%$ of patients with type 2 diabetes. Failure to recognise this can confound accurate blood pressure measurement and diagnosis of hypertension. New patients with diabetes should therefore be screened for an inter-arm difference using bilateral brachial blood pressure measurements.

\section{Funding body}

This study was funded by the Mid Devon Primary Care Research Group: PCT 0060. Christopher E Clark was funded by the Devon Workforce Planning Consortium/ Plymouth TPCT

\section{Ethical approval}

Ethical approval was obtained by the North \& East Devon Research Ethics Committee (2003/10/197) 


\section{Competing interests}

The authors have stated that there are none

\section{Acknowledgements}

We thank the patients of the Mid Devon Medical Practice, Wyndham House Surgery, and St Leonard's Medical Practice (an NHS R\&D practice) who gave their time. This study was originally proposed by $\operatorname{Dr} \mathrm{D}$ McLintock. $\mathrm{Dr} \mathrm{R}$ Powell gave statistical advice. We thank our nurses Jayne Fordham (Mid Devon), Trish Brown (Silverton), and Hilary

Clemoes (St Leonard's) for collecting the data.

\section{Discuss this article}

Contribute and read comments about this article on the Discussion Forum: http://www.rcgp.org.uk/bjgp-discuss

\section{REFERENCES}

1. Adler AI, Stratton IM, Neil HA, et al. Association of systolic blood pressure with macrovascular and microvascular complications of type 2 diabetes (UKPDS 36): prospective observational study. BMJ 2000; 321(7258): 412-419.

2. Clark CE, Powell RJ. The differential blood pressure sign in general practice: prevalence and prognostic value. Fam Pract 2002; 19(5): 439-441.

3. Clark CE, Powell RJ, Campbell JL. The interarm blood pressure difference as predictor of cardiovascular events in patients with hypertension in primary care: cohort study. J Hum Hypertens 2007; 21(8): 633-638.

4. Clark CE, Campbell JL, Powell RJ, et al. The inter-arm blood pressure difference and peripheral vascular disease: cross sectional study. Fam Pract 2007; 24: 420-426.

5. Williams B, Poulter NR, Brown MJ, et al. Guidelines for management of hypertension: report of the fourth working party of the British Hypertension Society, 2004-BHS IV. J Hum Hypertens 2004; 18(3): $139-185$.
6. Clark CE, Campbell JL, Evans PH, et al. Prevalence and clinical implications of the inter-arm blood pressure difference: a systematic review. J Hum Hypertens 2006; 20(12): 923-931.

7. English JA, Carell ES, Guidera SA, et al. Angiographic prevalence and clinical predictors of left subclavian stenosis in patients undergoing diagnostic cardiac catheterization. Catheter Cardiovasc Interv 2001; 54(1): $8-11$

8. Lane D, Beevers $\mathrm{M}$, Barnes $\mathrm{N}$, et al. Inter-arm differences in blood pressure: when are they clinically significant? J Hypertens 2002; 20(6): 1089-1095.

9. Kleefstra N, Houweling ST, Meyboom D, et al. Measuring the blood pressure in both arms is of little use; longitudinal study into blood pressure differences between both arms and its reproducibility in patients with diabetes mellitus type 2. Ned Tijdschr Geneeskd 2007; 151(27): 1509-1514.

10. Home P, Mant J, Diaz J, et al, on behalf of the Guideline Development Group. Management of type 2 diabetes: summary of updated NICE guidance. BMJ 2008; 336(7656): 1306-1308.

11. Gould BA, Hornung RS, Kieso HA, et al. Is the blood pressure the same in both arms? Clin Cardiol 1985; 8(8): 423-426.

12. Altman DG. Practical statistics for medical research. London: Chapman \& Hall, 1991

13. Aboyans V, Criqui MH, McDermott MM, et al. The vital prognosis of subclavian stenosis. J Am Coll Cardiol 2007; 49(14): 1540-1545.

14. Agarwal R, Bunaye Z, Bekele DM. Prognostic significance of between-arm blood pressure differences. Hypertension 2008; 51(3): 657-662.

15. Clark CE, Campbell J, Evans PH, et al. Detection of an interarm blood pressure difference in primary care diabetes care. Diabetic Medicine 2009; 24 (Suppl 1): 128.

16. Eguchi K, Yacoub M, Jhalani J, et al. Consistency of blood pressure differences between the left and right arms. Arch Intern Med 2007; 167(4): 388-393.

17. Waugh N. Reducing the rise in type 2 diabetes. Br J Gen Pract 2008; 58(553): 533-534. 